\title{
EL SIGNIFICADO DE LOS DETERMINANTES ESPAÑOLES
}

El sentido de una expresión léxica contiene toda la información necesaria para que, al combinarse con los otros constituyentes de la frase de la cual forma parte - siguiendo la información estructural-, se obtenga el sentido de la frase ${ }^{1}$.

Al escuchar un enunciado en nuestra lengua obtenemos de él

${ }^{1}$ Estoy empleando el término "sentido" dentro de la tradición que parte de Frege ("Über Sinn und Bedeutung") y que se ha mantenido en la semántica formal (por ejemplo, en la Gramática de Montague), pero con cierta visión modificada del concepto, como trataré de precisar. Llamo "sentido" tanto a los significados léxicos, parcialmente compartidos por los hablantes de una comunidad lingüística, como a los significados explícitos de las frases que se obtienen al combinar los primeros. Al funcionar estos significados dentro de un contexto - esto es, cuando el significado de la frase tiene una realidad y no se trata simplemente de una abstracción necesaria para su estudio-, puede ocurrir que se llegue a una significación bastante distinta del significado explícito de la frase, y que sea esta significación no explícita la que realmente funcione en condiciones normales - uno de los casos más claros lo constituyen las formas de cortesía; por ejemplo, cuando mediante una pregunta explícita se está haciendo en realidad una petición-. Esto es lo que, a mi parecer, podría motivar que se reservara el término "sentido" para el significado que se obtiene en el contexto, con lo que resulta más cercano al uso coloquial del término, como lo utilizan algunos autores - por ejemplo, Ducrot, en quien encontramos sens para referirse a la interpretación que incorpora la información que se obtiene de la situación de enunciación-. Sin embargo, puesto que el presente trabajo tiene sus antecedentes en otros en los que he utilizado "sentido" dentro de la tradición que parte de Frege, y como tal forma parte de una red de términos y conceptos, por razones prácticas, continuaré utilizándolo de igual manera. Por otra parte, considero que el sentido de un elemento léxico referencial es un conjunto de intersecciones de conjuntos. De estos últimos, cada uno estará definido mediante un rasgo del significado en cuestión (véase "Distintas dimensiones de la significación", ponencia que presenté en el II Coloquio Mauricio Swadesh [1990], próxima a publicarse en las actas correspondientes). 
un significado explícito; pero no se detiene aquí el fenómeno de la significación; éste se dispara hacia diversas dimensiones. No es mi propósito intentar aprehender en estas líneas toda la riqueza de la significación. Mi intención es acercarme a las bases estructurales de sus mecanismos en los determinantes ${ }^{2}$.

En este trabajo propongo una descripción del significado de los determinantes del español; en ella enfoco su funcionamiento en los siguientes procesos. Al obtener el sentido de una expresión lingüística, por él conocemos la extensión de la expresión; al interactuar el sentido con los datos que obtenemos del contexto - lingüístico y situacional - llegamos a identificar el referente de la expresión. El sentido interactúa con datos que provienen de distintos niveles de la enunciación ${ }^{3}$. Los implícitos que se desprenden del sentido nos remiten a las presuposiciones de la expresión, algunas veces directamente y otras veces a través de su interacción con la información referencial.

Por sentido de un elemento léxico entiendo el significado que comparten parcialmente todos los hablantes de una misma comunidad lingüística. Considero que mentalmente ${ }^{4}$ cada hablante tie-

${ }^{2}$ En una ponencia que leí en el II Coloquio M. Swadesh (cf. n. 1 supra), presento la repercusión de estos mecanismos en distintas dimensiones de la significación del habla coloquial y de algunos inicios de textos literarios.

${ }^{3}$ Como bien ha señalado Ducrot; véase Oswald Ducrot, "Analyse de textes et linguistique de l'énonciation" (en Ducrot et al, Les mots du discours, Les Editions de Minuit, Paris, 1980). Véase también la n. 1, supra.

${ }^{4}$ Parto del supuesto de que cada elemento o principio estructurante que utiliza un hablante para reproducir sus expresiones e interpretarlas, lo tiene registrado dicho hablante y que estos registros tienen una base de naturaleza neurofisiológica. Aunque se están realizando avances, cada vez más esclarecedores, en las investigaciones que se dirigen a encontrar correlatos entre realidades neurofisiológicas - principalmente de naturaleza eléctrica- y procesos lingüísticos, no estamos en posibilidades de describir la peculiaridad neurofisiológica particular y precisa que corresponde a cada fenómeno lingüístico. Cuando nuestro discurso se circunscribe en el objeto lingüístico, hablamos de entidades cuyo valor depende del propio modelo lingüístico - explícito como teoría o implícito como presupuestos teóricos-. Si nos apartamos lo suficiente del modelo propiamente lingüístico, para tener una visión más abarcadora, y nos interrogamos acerca de las realidades físicas que suponemos se relacionan con las entidades de nuestro modelo, hablaremos de entidades neurofisiológicas. Pero si en nuestra visión abarcadora no recurrimos a dicho cambio de perspectiva, entonces hablaremos de realidades psicológicas - aunque es cierto que existen otras posturas lingüísticas, en las cuales se considera que su modelo corresponde a un objeto que, mediante una idealización metodológica, resulta independiente de cualquier realidad psicológica- Cuando en el texto, con el fin de facilitar la expresión y siguiendo una tradición expresiva 
ne relacionado dicho sentido con el registro de la forma léxica.

$\mathrm{Al}$ analizar el sentido de un elemento léxico, si lo concebimos de manera aislada, difícilmente encontramos algunos datos con evidencias empíricas; por ejemplo, en el caso de los determinantes, sabemos que éstos no pueden llegar a tener un referente - entidad que nos permitiría tener un control de la adecuación empírica de nuestro análisis-; sin embargo, intervienen en el significado referencial de la frase sustantiva de la cual son constituyentes; esta intervención se realiza a través de su sentido. Pero, además de tener injerencia en la referencia de la frase, influyen en el sentido de ésta de una manera tal que no se tiene evidencia directa en la función referencial de la misma, sino en los posibles referentes de la oración de la cual forme parte dicha frase. Con el tipo de significaciones llamado presuposición sucede algo semejante. Por esto, al analizar el sentido de los determinantes, resulta indispensable tomar en cuenta su función dentro de unidades mayores. En resumen, al observar los fenómenos de significado en las unidades mayores en que un determinante aparece, se obtienen los datos necesarios para describir el sentido de éste. El sentido de un elemento se manifiesta de distintas maneras en estructuras de diferentes niveles de complejidad.

La delimitación de la extensión de una frase sustantiva se realiza de una manera que depende de su determinante:

1. mi pluma

2. una pluma

La extensión de 1, es decir, la representación de los objetos susceptibles de ser referentes de la frase ${ }^{5}$, se conforma partiendo del conjunto de objetos que tienen la propiedad de ser pluma y descartando, de este conjunto, a los que no tienen relación directa con el locutor ${ }^{6}$. Con 2, en cambio, la extensión de la frase se

- confieso que no podría asegurar que en tal uso la tradición que continúo sólo obedezca al plano de la expresión-, relaciono alguna entidad lingüística con registros o realidades "mentales", lo que está detrás es lo que en esta nota he querido resumir brevemente.

${ }^{5}$ La representación se considerará una entidad psíquica o una abstracción en el modelo, según el enfoque de nuestro objeto de estudio (cf. n. 4, supra). También podríamos hablar de la extensión en el mundo, haciendo abstracción de su realidad psicológico-perceptual.

${ }^{6}$ Utilizo "locutor" en el sentido de Ducrot ("La notion de sujet parlant", en Recherches sur la philosophie et le langage, t. 2, Université de Grenoble, 1982); es decir, para referirme al supuesto responsable del enunciado. 
mantiene idéntica a la extensión del sustantivo; la frase puede referirse a cualquiera de los objetos que tiene la propiedad de ser pluma; es decir que el determinante un no descarta, del conjunto de plumas, a ninguna; no exige que tengan alguna propiedad en particular, además de la requerida por el sentido del sustantivo: ser pluma - aun en los casos en que se puede deducir que en la intención del locutor hay una interpretación específica-. En general, podemos encontrar que los determinantes definidos delimitan extensionalmente las frases de las cuales son constituyentes, descartando a los miembros que no cumplan con alguna propiedad $^{7}$. La propiedad que exigen depende del sentido particular de cada determinante, como trataré de representar más adelante. Pero, dentro de ese sentido, todos los determinantes definidos tienen algo en común: comparten la misma manera de delimitar la extensión, descartando a algunos miembros de la extensión de los otros constituyentes de la frase. Los determinantes indefinidos, por su parte, mantienen la misma delimit .ción extensional a la que se llega al combinar los otros elementos de la frase sustantiva.

Como puede advertirse, utilizo el término extensión para referirme al ámbito de las posibilidades referenciales de una expresión lingüística; la extensión se desprende del sentido de la expresión. Con el término referente, en cambio, me refiero a la actualización de una de las posibilidades referenciales, con la cual relacionamos in facto la expresión al ser usada, y a la cual identificamos en este caso mediante la interacción del sentido y los datos del contexto.

Por razones metodológicas, al analizar el sentido, que es una unidad, lo trato como si estuviera constituido por dos aspectos: la manera de delimitar la extensión (como se ejemplificó con 1 y 2, supra) y la mención:

2. una pluma

3. la pluma

Mediante 2 mencionamos a uno de los miembros pertenecientes a la extensión de la frase; aunque con la misma frase 2 podamos referirnos, bien a uno solo de los miembros, o bien a todos ellos:

7 Véase J. García FAJARdo, "El sentido en la función referencial de frases sustantivas del español', NRFH, 37 (1989), 19-26. 
4. Acabo de comprar una pluma

5. Una pluma funciona con tinta

Con 3 mencionamos la extensión de la frase como conjunto; el conjunto al que hagamos referencia puede ser unimembre o plurimembre. Del contexto podemos obtener los datos para conocer su pluralidad o singularidad:

6. Se me perdió la pluma que me regalaste

7. La pluma es un artefacto muy útil

Los determinantes indefinidos mencionan la extensión mediante sus miembros y los definidos la mencionan como conjunto. Con esto quiero decir que estoy considerando dentro de la mención esa parte del significado que tiene la propiedad de imprimir en la frase el sentido individual o el sentido de conjunto. La diferencia entre estos sentidos se hace patente al contrastar los posibles referentes de las oraciones:

8. Una bugambilia da flores de distintos colores

9. La bugambilia da flores de distintos colores

Cada una de estas oraciones puede referirse a todas las bugambilias del universo; esto es, entre otras posibles interpretaciones, puede tener la genérica. Es ésta la interpretación que me interesa enfocar aquí para mostrar el fenómeno en cuestión ${ }^{8}$. Dentro de la interpretación indicada, con 8 entendemos que en cada planta la floración es multicolor ${ }^{9}$, mientras que con 9 podemos entender esto mismo, o bien, que el conjunto de bugambilias del universo es multicolor, pero quizás con la floración de cada planta de un solo color. Esta segunda interpretación no es posible con 8. El determinante indefinido origina en la frase sustantiva el sentido individual, mientras que el definido origina el sentido de con-

${ }^{8}$ En J. García Fajardo, El sentido de los sintagmas nominales'y los tipos de predicación (I.N.A.H., México, 1985; en adelante: 'SSNyTP), se describen las distintas interpretaciones que pueden tener las frases con artículo indefinido, incluyendo aquéllas mediante las cuales se puede hacer referencia a un subconjunto del género.

${ }^{9}$ Otra de las posibles ambigüedades de estas oraciones se basa en el mecanismo de la combinación de un sustantivo plural y un complemento (véase la n. 10, infra). Mediante esta ambigüedad podremos entender que cada flor es de varios colores o que el conjunto de ellas lo es. 
junto. Al tener la frase un sentido individual, el significado del predicado se relacionará con la representación de cada miembro de los objetos o individuos a los cuales se refiere: 'dar flores de distintos colores' se relacionará con cada 'bugambilia'. Si la frase tiene sentido de conjunto, el significado del predicado se relacionará con el conjunto como unidad, dando como resultado una estructura semántica; y se relacionará también con cada miembro de ese conjunto, produciendo otra estructura semántica. De esta manera obtenemos, con las frases de sentido individual, la predicación individual únicamente; y, con las frases de sentido de conjunto, la predicación de conjunto y la predicación individual: dos estructuras semánticas de la oración producidas por el sentido que la frase sustantiva obtiene del significado del determinante. El mismo contraste de interpretaciones obtenemos con:

10. Toda bugambilia da flores de distintos colores

11. Las bugambilias dan flores de distintos colores

10 sólo tiene la predicación individual; 11 tiene la predicación de conjunto y la individual ${ }^{10}$.

A continuación ofrezco, en primer lugar, una descripción del sentido de algunos determinantes del español, dividida en dos subapartados, por razones de claridad. En el primer subapartado aparece el sentido en la perspectiva de delimitador de la extensión; en el segundo, aparece el sentido desde el enfoque al que he llamado "mención". Posteriormente, pretenderé mostrar cómo es que a partir del sentido, ya sea desde su enfoque de delimitador o de mención, se llega a la referencia (apartado 2), a los implícitos (apartado 3) y a algunos efectos de la significación no explícitos (apartado 4). Finalmente, bosquejaré una formalización del sentido de cada determinante considerado (apartado 5), con el solo propósito de mostrar que las realidades de la significación que analizo en el presente trabajo corresponden a esa parte del significado que es posible aprehender formalmente ${ }^{11}$.

${ }^{10}$ En 11 el sentido de conjunto está doblemente motivado: por el determinante definido de la frase sustantiva y por su marca de plural. El sentido de conjunto se origina en el sentido de los determinantes definidos, en la marca de plural y en la conjunción de frases sustantivas. Véanse J. García FA. JARDO, SSNyTP y Un estudio del significado. 'Presupuestos, principios y desarrollo, I.N.A.H., México, 1989.

${ }^{11}$ iGracias a Dios siempre habrá realidades de la significación que sigan volando libremente! Éstas seguirán motivándonos. 


\section{Sentido}

\subsection{Delimitación de la extensión}

Las dos maneras de delimitar la extensión, una correspondiente a los determinantes definidos, con descarte de miembros, y otra correspondiente a los indefinidos, sin descarte de miembros, fueron expuestas en un trabajo anterior ${ }^{12}$, por lo que en este apartado trataré de ser muy breve, anotando sólo los datos necesarios para el presente desarrollo.

El artículo definido delimita extensionalmente la frase sustantiva descartando a los miembros que no pertenecen al universo del discurso. Cuando en el universo del discurso no encontramos ningún miembro del conjunto delimitado extensionalmente por el sentido de los otros constituyentes de la frase (el sustantivo y sus modificadores adjetivales) - esto es, cuando no ha sido mencionado anteriormente dentro del discurso ni se encuentra en la inmediatez del contexto situacional-, y dicho universo no resulta pertinente para identificarlo, la representación universal fungirá como universo del discurso ${ }^{13}$. Sólo en el último caso, la extensión de toda la frase sustantiva coincidirá con la extensión que se obtiene sin el determinante.

Una frase como el libro tendrá, entonces, como extensión al conjunto de libros que haya sido mencionado anteriormente -o que se encuentre próximo al acto de la enunciación-, o que pertenezca al universo por la delimitación que, en el discurso, se haya hecho de una época, de un tema, de una editorial, etcétera. Dicho conjunto puede ser unimembre, cuando se ha hablado previamente, por ejemplo, de el último libro de algún escritor; o puede ser plurimembre, como cuando se ha hablado del conjunto de libros de alguna editorial. Cuando no se ha delimitado el conjunto de libros dentro del universo particular del discurso en el que se inserta la frase el libro, la representación universal de los libros pasará a pertenecer al universo del discurso, como un conjunto plurimembre, y la frase tendrá una interpretación genérica.

El determinante demostrativo este delimita extensionalmente la frase sustantiva descartando a los miembros que no tienen la

12 Véase la n. 7.

13 Actualmente se encuentra en preparación un trabajo sobre la delimitación del universo del discurso desde la perspectiva de los tipos de predicación. Por el momento, es importante tener en cuenta que, a través de las frases sustantivas, el discurso mismo, al irse desenvolviendo, va delimitando su propio universo. 
propiedad de estar en relación de primera cercanía contextual en las dimensiones del texto o del contexto situacional. En el primer caso, quedará delimitado el último subconjunto mencionado, incluido en el conjunto delimitado por los otros constituyentes de la frase sustantiva. En el segundo caso, se delimitará el subconjunto más cercano, en el espacio, al lugar de la enunciación.

El determinante posesivo delimita extensionalmente descartando a los miembros que no están en relación directa con la persona a la que corresponde el posesivo: con el locutor (el responsable de la enunciación fuera de la ironía y que suele coincidir con el hablante, cuando éste no está haciendo una cita en estilo directo), en el caso de $m i$.

El artículo indefinido no descarta, de la extensión delimitada por los otros constituyentes de la frase, a ningún miembro. Así, para pertenecer a la extensión de un libro, sólo se requiere tener la propiedad de ser libro; todos aquellos objetos que la tengan cabrán dentro de la extensión de la frase.

Todo delimita extensionalmente sin descartar miembros. En todo libro, la extensión está formada por todos los objetos con la propiedad mencionada en el sustantivo.

Cada delimita extensionalmente sin descartar miembros. Cada libro tendrá, como extensión, a todos los objetos que tengan la propiedad mencionada en el sustantivo.

Algún delimita extensionalmente sin descartar miembros. $A l$ gún libro tiene, por extensión, a todos los libros.

\subsection{Mención}

Con los determinantes definidos mencionamos un conjunto equivalente al delimitado extensionalmente. En cambio, con los determinantes indefinidos mencionamos a los miembros pertenecientes a la extensión. Con un mencionamos a uno de los miembros pertenecientes a la extensión. Con todo, a los miembros de la extensión, enfocando la totalidad. Con cada, a los miembros de la extensión, enfocando la individualidad. Con algún, a uno de los miembros pertenecientes a la extensión, enfocando su no especificidad.

\section{RefERENCIA}

Por la función referencial, en general, el sentido de toda frase sustantiva interactúa con los datos que tenemos del contexto - lin- 
güístico y extralingüístico-, centrándose en él. Con las frases cuyo determinante es definido, puesto que en su sentido se mencionan factores situacionales específicos, la interacción se dirige a ellos. Es decir, en la función referencial de frases de artículo definido o de determinantes demostrativo o posesivo, se aplica la mención al contexto situacional. De esta manera, la "búsqueda" del conjunto cuyos miembros tienen las propiedades mencionadas en la combinación entre el sustantivo y sus adjetivos, se dirige: hacia el universo del discurso, si el determinante es un artículo definido; hacia el contexto, siguiendo una línea, desde el lugar o desde el momento de la enunciación hacia la distancia (espacial o temporal), para encontrarse con su subconjunto más cercano, cuando el determinante es el demostrativo este; o hacia un subconjunto suyo que tenga relación directa con el locutor si el determinante es el posesivo mi.

Con el determinante indefinido un, se aplica la mención a la extensión mediante dos caminos; en uno de ellos, se aplica una sola vez (y nos refériremos a un solo objeto, como sería la interpretación más usual de estoy escribiendo un artículo); en el otro camino, se aplica recursivamente hasta agotar la extensión (como en la interpretación genérica de un perro tiene cuatro patas).

Con todo y con cada, se aplica la mención a la extensión hasta agotarla. Con algún, se aplica la mención a la extensión y no se llega a un referente específico.

\section{ImPLícitos}

Del sentido de las frases sustantivas se desprenden algunas implicaciones, de la misma forma en que una conclusión se desprende de sus premisas y corresponde al consecuente de un condicional tautológico. Otros implícitos no parecen ser implicaciones formales, en el uso técnico del término, puesto que no parecen desprenderse del sentido de una manera que siga las leyes de inferencia de alguna lógica formalizada, sin ningún salto. Se desprenden más bien del hecho de seleccionar ese sentido y no otro ${ }^{14}$. De todos

${ }^{14}$ Los dos implícitos del determinante $e l$ son un buen ejemplo de implicación formal estricta. Sin embargo, los implícitos del determinante un resultan, no de la de la noción aislada de 'ser miembro de', sino del uso de ella. Como bien sabemos, en matemática, decir que un individuo es miembro de un conjunto no implica que en dicho conjunto haya otros miembros. Sin embargo, en lengua natural, el hecho de seleccionar un y no $e l$ parece expresar que el conjunto correspondiente no es unimembre. 
estos implícitos -implicaciones estrictas o no-, algunos se originan en el sentido del determinante -o de su uso-. De acuerdo con las descripciones del sentido de los determinantes que he propuesto (apartados 1.1 y 1.2), los implícitos que se basan en las frases sustantivas correspondientes serían los siguientes:

$e l:$ 1) En el universo del discurso, la extensión de la frase sustantiva es idéntica a la extensión que se obtiene al combinar los constituyentes de la misma frase a excepción del determinante. 2) No existen, en el universo del discurso, otros miembros del conjunto mencionado en el sustantivo (y sus adjetivos) más que aquéllos (o aquél) a los que se hace referencia con la frase completa (para no perderse en éstos que parecen juegos de palabras, puede acudirse únicamente a las abreviaturas):

1) U.D.: Ext (F. sust.) = Ext (F. sust.-Det.)

2) U.D.: - Vx ((F. sust.-Det. $) x \&-(\operatorname{Ref}($ F. sust. $) x))$

este: 1) La extensión de la frase sustantiva es menor que la extensión que resulta de combinar los constituyentes de la frase excluyendo al determinante. 2) Existen otros miembros del conjunto mencionado en el sustantivo (y sus adjetivos) además de aquéllos (o aquél) a los que se hace mención con la frase completa. 3) En relación de primera cercanía contextual con respecto al lugar o momento de la enunciación, la extensión de la frase sustantiva es idéntica a la extensión que resulta de combinar los constituyentes de la frase excluyendo al determinante. 4) No hay otros miembros del conjunto mencionado mediante el sustantivo (y sus adjetivos) que estén en relación de primera cercanía contextual, además de aquél(los) al(os) que se hace referencia con la frase completa:

1) Ext (F. sust.) $<$ Ext (F. sust.-Det.)

2) $\operatorname{Vx}($ Ext (F. sust.-Det.)x \& -(Ext (F. sust.)x))

3) R.G. $1^{\mathrm{a}}$ : Ext (F. sust.) = Ext (F. sust.-Det.)

4) $-\operatorname{Vx}\left((\right.$ F. sust.-Det. $) x \&\left(\right.$ R.C. a $\left.^{\text {a }}\right) x \&-(\operatorname{Ref}($ F. sust. $\left.) x)\right)$

$m i$ : 1) y 2) son iguales a las implicaciones 1) y 2) que se originan con este. 3) En relación directa con el locutor, la extensión de la frase sustantiva es idéntica a la extensión que resulta de combinar los constituyentes de la frase excluyendo al determinante. 4) No existen otros miembros del conjunto mencionado mediante el sustantivo (y sus adjetivos) que estén en relación directa con el locutor, además de aquél(los) a(los) que se hace referencia con la frase completa: 
1) $=$ 1) este

2) $=$ 2) este

3) R.L.: Ext (F. sust.) = Ext (F. sust.-Det.)

4) -Vx ((F. sust.-Det.) $x$ \& (R.L.) $x \&-(\operatorname{Ref}(F$. sust. $) x))$

un: 1) En la extensión de la frase sustantiva existe más de un miembro. 2) La referencia de la frase sustantiva es menor que la extensión de la frase sustantiva, si en el primer camino de la función referencial (sin recursividad en la aplicación de la mención) se llega a la referencia; y son idénticas la referencia y la extensión si en el segundo camino se llega a la referencia (con recursividad). 3) Si el primer camino hacia la función referencial llega al referente de la frase, entonces existen otros miembros del conjunto mencionado en el sustantivo (y sus adjetivos) además de aquel sobre el que caiga la referencia. Si el segundo camino hacia la función referencial llega al referente, entonces no existen otros miembros del conjunto mencionado por el sustantivo y sus adjetivos además de aquellos mencionados con la frase completa.

1) Vx Vy $(($ Ext (F. sust. $)) x \&($ Ext (F. sust. $))$ y \& $x \neq y$

2) -Rec: Ref (F. sust.) < Ext (F. sust.)

Rec: $\operatorname{Ref}(F$. sust. $)=$ Ext (F. sust.)

3 ) Rec: Vx ((F. sust.-Det.)x \& -(Ref (F. sust.)x))

Rec: -Vx $((F$. sust.-Det. $) x \&-(\operatorname{Ref}(F$. sust. $) x))$

todo: 1) La extensión de la frase sustantiva es igual a la extensión que se obtiene de combinar los constituyentes de la frase sustantiva a excepción del determinante. 2) No existe(n) otro(s) miembro(s) del conjunto mencionado en el sustantivo (y sus adjetivos) más que aquéllos a los que se hace referencia mediante la frase sustantiva completa.

1) $\operatorname{Ext}$ (F. sust.) = Ext (F. sust.-Det.)

2) $-\operatorname{Vx}((F$. sust.-Det.) $x \&$-(Ref (F. sust.) $x))$

cada: 1) y 2) son iguales a las implicaciones 1) y 2) de todo.

algún: 1) es igual a la implicación 1) de un. 2) y 3) son iguales, respectivamente, a las implicaciones 2) y 3) sin recursividad de un.

\section{Efectos de Significación NO EXPLí́cita}

El sentido de los determinantes, a través de sus implícitos, genera distintos efectos de significación no explícita. En este trabajo 
me limito a ilustrar sucintamente la función del sentido en unos cuantos ejemplos ${ }^{15}$.

Del implícito 2) del artículo definido, llegamos a saber que no existen otros individuos (objetos o hechos), en el universo del discurso, con las propiedades mencionadas en el núcleo de la frase (más sus adjetivos), además de aquel(los) en quien(es) cae la referencia. De esta manera, si la referencia cae en un solo individuo, y de esto nos enteramos, por ejemplo, por lo que se predica de él, entonces concluimos que ese individuo es único en el sentido de que no existe otro - al menos en el universo del discursoque posea las mismas cualidades mencionadas en la frase. De aquí surge la "presuposición de unicidad" 16 . Así es como por la expresión:

\section{Al dentista del pueblo lo hospitalizaron ayer}

podemos llegar a conocer que en el pueblo al que se hace referencia sólo hay un dentista, aunque no esté dicho explícitamente. Mediante este tipo de presuposición obtenemos información sobre el contexto de referencia. Es posible que esa información resulte nueva - susceptible de constituir un conocimiento.

Por la delimitación de la extensión, una frase iniciada con artículo definido puede tener como extensión a un individuo que no haya sido mencionado previamente y que no se encuentre presente en el contexto situacional. Esto tiene como consecuencia que la mención del referente mediante la frase con artículo definido

${ }^{15}$ En el trabajo presentado para el II Coloquio M. Swadesh (cf. n. 1), describo distintas clases de significaciones no explícitas, generadas por el sentido de los determinantes a través de sus implícitos.

${ }^{16}$ Tal y como se describió en J. García Fajardo, "El sentido de conjunto y un tipo de presuposición" (en Homenaje a Jorge A. Suárez. Lingüística americana, ed. de B. Garza Cuarón y P. Levy, El Colegio de México, México, 1990). Este trabajo se centró en mostrar que no era necesario añadir a la descripción del significado del artículo definido un contenido ad hoc para obtener de él la presuposición de unicidad, sino que, como puede constatarse en la presente exposición, la misma descripción realizada para dar cuenta del sentido de conjunto, que surge del artículo, al combinarse con la información de la referencia, conduce a dicha presuposición. Y, además, de esta manera, la descripción del significado del artículo resulta adecuada, tanto para las frases que se refieren a un solo individuo, como para las frases genéricas (mientras que las descripciones que añadían un contenido ad hoc para dar cuenta de la presuposición de unicidad resultaban inadecuadas para dar cuenta de las interpretaciones genéricas). 
ocurra por primera vez al mismo tiempo que se ofrece la presuposición de unicidad. Como hemos visto, esta presuposición surge del hecho de combinar el sentido del determinante definido con la información de que se está haciendo referencia a un solo individuo. En el escucha para quien esta información no provenga de una representación previa del referente, sino que se infiera del contenido de una predicación como la del ejemplo precedente, la presuposición llega a constituir una información nueva.

De los implícitos de un determinante demostrativo como este obtenemos con menos facilidad información nueva sobre el contexto de referencia. Por estos implícitos sabemos que en el universo del discurso existen otros objetos (individuos o hechos) con las propiedades mencionadas en el núcleo de la frase (y sus adjetivos) además de aquel en que cae la referencia; pero que es el único que está en relación de primera cercanía contextual con el lugar o el momento de la enunciación. Sin embargo, lo más común es que el destinatario de la expresión no desconozca estas propiedades de relación, por estar implicado en ellas, por lo que, con frecuencia, no resultan información nueva. Aunque puede suceder que el locutor, hablando por teléfono, diga algo así como:

\section{Hasta luego, voy a leer este libro}

cuando no se ha mencionado previamente ninguno, con lo cual, la persona que está al otro lado de la comunicación se enterará de que el locutor tiene un libro cerca. O, por el implícito 2) del demostrativo este, podemos saber que el locutor tiene otra(s) sobrina(s), al escucharle una expresión como:

\section{Esta sobrina vive en San Juan del Río}

Otra información que puede obtenerse mediante las implicaciones del demostrativo es la de distancia afectiva:

15. No sé de dónde le salen ESASS ideas a Elena. Ella no fue educada de ese modo.

Carlos Fuentes, Las dos Elenas (las mayúsculas cursivas son mías)

Por supuesto, se hablaría de "estas ideas" - y no de "esas ..." -, en ese contexto, para referirse a las que públicamente se reconocen como centrales del paradigma de educación del(a) locutor(a). 
Del implícito de que no existe otro individuo en relación directa con el locutor, desprendido del sentido de $m i$, con la siguiente expresión interpretamos que su locutor tiene sólo un hijo -o, claro, que ése es el único que pertenece al universo del discurso en turno-:

\section{Mi hijo tiene veinte años}

En un inicio de texto literario, al no disponer de previo universo del discurso, el sentido mismo del posesivo, que contiene la noción de relación directa con el locutor, tiene el efecto de ubicar el centro de la narración, desde el cual se van a exponer los hechos:

17. Mi primera mujer, la primera que desnuda conocí, llamábase Faustina.

Juan de la Cabada, Mi primera mujer.

Por los implícitos del artículo un es que una jovencita puede incomodarse si su novio la presenta diciendo:

18. - Te presento a una novia.

${ }^{17}$ Considero que la presuposición de existencia de la referencia no se desprende de la frase sustantiva, sino de la enunciación de una predicación. Por esto, en las fórmulas de los determinantes no aparece el contenido correspondiente a dicha presuposición. En ellas empleo algunas constantes: el subscrito " " $T$ " indica que se trata de una predicación que sigue los mecanismos de los tipos de predicación (individual o de conjunto). La necesidad de este símbolo se expuso en un trabajo anterior (Un estudio del significado. Presupuestos, principios y desarrollo). "U.D." representa al conjunto del universo del discurso. Los símbolos " $D_{1 \mathrm{aE}}$ " y " $\mathrm{R}_{\mathrm{LOC}}$ " representan, respectivamente, "estar en primera cercanía con respecto a la enunciación" y "estar en relación directa con el locutor". El símbolo * tiene un efecto formal idéntico a la sustitución de la variable ligada por un cuantificador universal, con la única restricción expresada en la fórmula: pertenecer a $P$. Éste es el efecto requerido en uno de los caminos de la función referencial de un: aplicar la mención (x) a la extensión (P) recursivamente, hasta agotar dicha extensión. En estas formalizaciones he intentado recoger los aspectos del sentido de los determinantes que analizo en el texto. Quedan, sin embargo, algunos pendientes, como la incógnita de decidir sobre la representación de la diferencia entre un (en su interpretación no genérica) y algún; en el sentido de este último he considerado un enfoque a la no especificidad; sin embargo, estoy consciente de que el concepto mismo de "enfoque" requiere esclarecimiento. 


\section{FORMALIZACIÓN DEL SENTIDO ${ }^{17}$}

$\begin{array}{ll}\text { el } & \lambda \mathrm{P} \lambda \mathrm{Q}\left(\mathrm{Q}_{\mathrm{T}}(\mathrm{C} / \mathrm{C}=\mathrm{P} \cap \mathrm{U} . \mathrm{D} .)\right. \\ \text { este } & \lambda \mathrm{P} \lambda \mathrm{Q}\left(\mathrm{Q}_{\mathrm{T}}\left(\mathrm{C} / \mathrm{C}=\mathrm{P} \cap \mathrm{D}_{1 \mathrm{aE}}\right)\right. \\ m i & \lambda \mathrm{P} \lambda \mathrm{Q}\left(\mathrm{Q}_{\mathrm{T}}\left(\mathrm{C} / \mathrm{C}=\mathrm{P} \cap \mathrm{R}_{\mathrm{LOC}}\right)\right.\end{array}$

$u n$

$$
\begin{aligned}
& \left.\left({ }^{*} \mathrm{x} / \mathrm{x} \in \mathrm{P}\right)\right) \\
& \lambda \mathrm{P} \lambda \mathrm{Q}\left(\mathrm{Q}_{\mathrm{T}}\right. \\
& (x / x \in P))
\end{aligned}
$$

$\begin{array}{ll}\text { todo } & \lambda \mathrm{P} \lambda \mathrm{Q} \wedge \mathrm{x}\left(\mathrm{P}(\mathrm{x}){ }^{\Omega} \mathrm{Q}_{\mathrm{T}}(\mathrm{x})\right) \\ \text { cada } & \lambda \mathrm{P} \lambda \mathrm{Q}\left(\mathrm{Q}_{\mathrm{T}}\left({ }^{*} \mathrm{x} / \mathrm{x} \in \mathrm{P}\right)\right) \\ \text { algún } & \lambda \mathrm{P} \lambda \mathrm{Q}\left(\mathrm{Q}_{\mathrm{T}}(\mathrm{x} / \mathrm{x} \in \mathrm{P})\right)\end{array}$

Puede notarse que, desde la perspectiva asumida en este trabajo, las implicaciones derivadas del sentido de los determinantes interactúan con algún factor interno de la enunciación - como el hecho de haber sido dicho- o con la información sobre el referente; y de esta manera surge alguna presuposición. En consecuencia, las presuposiciones - por lo menos las relacionadas con los determinantes - no están contenidas en el sentido de las frases o de las oraciones ${ }^{18}$, pero sí tienen su base en él. Así, puede explicarse el hecho de que las oraciones afirmativas y sus correspondientes negaciones mantengan la misma presuposición ${ }^{19}$; y,

${ }^{18}$ A diferencia de la concepción de Russell (véase " On denoting", Mind, 14, 1905, 479-493). En este aspecto me alejo de la formalización que ofrece Montague para el significado del determinante the (véase "The proper treatment of quantification in ordinary English', en R. Thomason, Formal philosophy. Selected papers of Richard Montague, Yale University, New Haven, 1974).

19 Tal como Frege mostró, originando un historial de polémicas en la tradición filosófica (véase "Sobre sentido y referencia", en G. Frege, Estudios sobre semántica, Ariel, Barcelona, 1973, pp. 49-84). Recordemos que si se considera que las presuposiciones están contenidas en el sentido, la negación formal de las proposiciones de las que formarían parte no correspondería a las intuiciones que los hablantes tienen de su lengua, pues esas negaciones formales estarían constituidas por una disyunción, uno de cuyos miembros representaría la presuposición. Esto - entre otras cosas - ha conducido a considerar las presuposiciones como una significación no explícita que pertenece a un nivel distinto del sentido. En la descripción que aquí propongo, se especifica la vinculación entre las presuposiciones relacionadas con los determinantes y el sentido. 
al mismo tiempo, queda descrito el mecanismo mediante el cual surge ésta a partir del sentido.

De esta manera he intentado mostrar cómo el sentido de los determinantes, tal y como aquí se describe, interviene en la función referencial del uso de las frases - mediante la delimitación extensional - y en la función referencial de las oraciones - mediante los tipos de predicación-, así como en la generación de significaciones no explícitas.

Josefina García Fajardo El Colegio de México 